\title{
THE COMPLEMENTARY ROLE OF THE RAMAN MICROSPECTROSCOPY TO THE OXIDATIVE STRESS ASSAYS IN THE NEONATAL SYNAPTOSOMES CHARACTERIZATION
}

\author{
VLAD-ALEXANDRU TOMA \\ IOANA BREZEȘTEAN ${ }^{\mathrm{a}, \mathrm{d}}$, BOGDAN DUME ${ }^{\mathrm{b}}$, \\ IOANA ROMAN ${ }^{\mathrm{c}}$, IOAN TURCU ${ }^{\mathrm{a}^{*}}$
}

\begin{abstract}
Raman microspectroscopy was tested as an alternative/ complementary method for biochemical evaluation of the synaptosomes obtained from neonatal rat brain prenatally exposed to sodium valproate and treated with allicin. Spectrophotometric assays of several oxidative stress markers (catalase, superoxide dismutase, total thiols) and acetylcholine esterase activity revealed the redox balancing function and pro-cholinergic effect of the allicin as compared to the valproate effect. Raman evaluation showed no significant changes in our experimental conditions. Different concentrations and volumes of the synaptosomes vesicles must be tested for the optimal Raman examination of these purified synaptosomes.
\end{abstract}

Keywords: Raman, synaptosomes, brain, vesicles, sensitive method, redox status

\section{INTRODUCTION}

The structural diversity of the nervous system frequently needs multidisciplinary perspectives in order to evaluate a phenomenon in a more accurate manner. As described by Frisch et al. [1], prenatally exposure of Wistar rats to high dosages of sodium valproate induced in newborn individuals

a National Institute for Research and Development of Isotopic and Molecular Technologies, Donath str. No. 67-103, 400293 Cluj-Napoca, Romania

b Babeș-Bolyai University, Faculty of Biology and Geology, Clinicilor str. No. 5-7, 400006 Cluj-Napoca, Romania

c National Institute of Research and Development for Biological Sciences, Institute of Biological Research, Republicii str. No. 48, 400015 Cluj-Napoca, Romania

d Babeş-Bolyai University, Faculty of Physics, Kogalniceanu str. No.1, 400084, Cluj-Napoca, Romania

* Corresponding author: ioan.turcu@itim-cj.ro 
cognitive teratogenicity that might be associated with the autism spectrum disorders (ASDs). ASDs were characterized by redox imbalance based on the down-regulation of the factor erythroid-derived 2-like 2 (Nrf2). Alongside, in ASDs were observed arterial hypertension by endothelial NO synthetase (eNOS) deficiency, DNA hyperacetylation, neuronal hyperconnectivity with increased ATP cycle, and synaptic deficiency of postsynaptic density protein 95 (PSD-95). The allicin (which can be easily obtained from garlic and onion) acts as an antioxidant, NRF2 activator, increases the concentration of the S-allyl-mercapto-glutathione [2] in brain, liver, or plasma and increases the NO concentration in blood. Until now, ASDs are established just after the appearance of the symptoms at 2-3 years old. The context of ASDs manifestation greatly restricts the investigation methods, many of these being invasive or applied too late. As a prototype idea, the investigation of the synaptic feature with very sensitive methods could form a basis for the early identification of an ASDs-related pattern in newborns. The synaptosomes are vesicles that contain pre/post-synaptic densities, mitochondria, and whole synaptic neurochemical complex. These vesicles, like the exosomes, pass over the cellular membranes and can be identified in the bloodstream [3]. Based on these pieces of evidence, we tested as a first step, the Raman microspectroscopy on the neonatal brain synaptosomes associated with redox-cholinergic status evaluation.

Raman microspectroscopy is a noninvasive and nondestructive labelfree technique that uses the inelastically scattered light to rapidly provide biochemical and structural information from molecules $[4,5]$. Each molecule is characterized by a unique "spectral fingerprint" which comprises its vibrational pattern dependent on its constituent chemical bonds and structure [5]. Recently, the Raman technique has been recognized as a very powerful analytical tool to help advance the field of neuroscience [6]. It has been used to characterize fundamental biological molecules in cells $[4,7]$ to discriminate normal cells from cancer cells on the basis of their biochemical differences $[4,8,9,10]$ and to detect protein misfolding and aggregation, key modifications that take place in the early stage of neurodegenerative diseases $[5,10,11$, 13]. To further enhance its helpfulness, Raman microspectroscopy has been multiplexed with other analytical methods (such as advanced statistical techniques) to shed light on the relative abundance of various molecules in the brain [13].

In this work we employed Raman spectroscopy for the rapid characterization of synaptosomes (SYN) prepared from neonatal Wistar rat brains and as a detection tool to identify the vibrational changes induced into the SYN samples treated with either sodium valproate (SYN@VAL),-allicin (SYN@ALI) or both (ALI@SYN@VAL). Mizuno et al. [14] reported an early 
study focused on the use of Fourier Transform (FT) Raman spectroscopy in monitoring the relative changes of proteins and lipids. Moreover, the study was able to reveal the structural changes and the component content ratio from the synaptosomal fraction of the rat brain. Ajito et al. [7] applied a laser trapping technique combined with near-infrared (NIR) Raman spectroscopy (LTRS) for the analysis of SYN isolated from the rat brain, dispersed in the phosphate buffer solution [7, 15]. Their results indicated that the laser-trapped synaptosomes include some types of lipids and proteins. In another study [16], the same group describes in situ detection of glutamate released from a single SYN using LTRS. As far as we know no Raman spectroscopic investigations were reported until now on SYN@VAL, SYN@ALI, and ALI@SYN@VAL.

\section{RESULTS AND DISCUSSION}

The oxidative stress measurements have revealed that valproate treatment in adults decreased $(P<0.01)$ the catalase (CAT) activity (Fig. 1a) in newborns as well as allicin administration $(P<0.05)$. Combined therapy normalized the CAT activity as compared to Control but remained slightly decreased $(P<0.05)$. Superoxide dismutase (SOD) (Fig. 1b) activity was unchanged after valproate exposure but allicin administration slightly increased the enzyme activity $(P<0.05)$. The oxidative stress enzymes and other nonenzymatic antioxidants such as reduced glutathione (GSH) or ascorbate cannot be described as standard parameters which are in direct relationship with oxidative stress increasing or decreasing. Our data suggest firstly that CAT decreasing was not associated with SOD decreasing and secondly, the antioxidants such as allicin reduced the CAT activity and induced a high activity of the SOD, according to our previous results [17]. As was noticed by Loyd [18], valproate administration reduced the activity of several antioxidants such as CAT, SOD, or peroxidase via the NRF2 down-regulation effect. Our results partially confirm these findings by decreasing the CAT activity whereas SOD remained unchanged.

The nonenzymatic antioxidant potential of the synapses was evaluated by measuring the concentration of the total - $\mathrm{SH}$ groups (Fig. 1c). Valproate administration did not change the level of the thiols in synaptosomes while allicin treatment significantly decreased $(P<0.001)$ the total $-\mathrm{SH}$ concentration. Decreasing of the -SH groups after allicin administration was noticed in blood and liver by Rabinkov et al. [19] and confirmed by our previous results [17]. Briefly, the allicin reacts with GSH and forms a new 
compound, S-allyl-mercapto-glutathione which is not detected by Ellman reaction. Based on this evidence, although the concentration of $\mathrm{GSH}$ and -SH groups decreased after allicin treatment, a new thio-compound, S-allylmercapto-glutathione $[17,19]$ restore the thio-compounds pool. These findings demonstrated that prenatally administration of sodium valproate will expose the newborns to oxidative stress by reducing the antioxidant enzymes. In contrast, prenatally administration of allicin maintained or increased the antioxidant capacity in the synaptic complex. Various relationships between oxidative stress and chemical signaling of the neurons were described in literature $[20,21]$. Our experimental context was correlated to the valproate signaling effect by increasing $y$-aminobutiric acid (GABA) concentration [22]. Since GABA is an inhibitory molecule, we evaluate the opposite effect by measuring the acetylcholine esterase (AChE) activity in the purified synaptosomes (Fig. 1d).

Allicin administration slightly increased $(P<0.05)$ AChE whereas valproate significantly decreased the activity of this enzyme $(P<0.001)$ and the combined therapy normalized the AChE activity as compared to Control. Our results confirm several previous findings of the stimulated cholinergic effect of the allicin [23] and argue the positive cognitive effect of the allicin consumption [24]. These results were then associated with Raman microspectroscopy in order to evaluate the complementary role of the spectroscopic methods in the synaptosome characterization. The Raman results are shown in Fig. 2. Very similar spectra are the ones of the control sample and the SYN@ALI sample, while SYN@VAL and ALI@SYN@VAL also present identical features.

The Raman spectra of SYN and SYN@ALI samples are dominated by the sharp, intense peak at $669 \mathrm{~cm}^{-1} / 678 \mathrm{~cm}^{-1}$ which can be assigned to C-S stretching mode or to deformations in cysteine [5]. Another welldefined but low-intensity Raman band is at $705 \mathrm{~cm}^{-1} / 714 \mathrm{~cm}^{-1}$, coming from cholesterol [5]. The same authors assign the Raman bands located in the spectral range $1480-1554 \mathrm{~cm}^{-1}$ to $\mathrm{C}-\mathrm{H} / \mathrm{C}=\mathrm{N}$ deformation and to amide II, while the band at $1716 \mathrm{~cm}^{-1} / 1721 \mathrm{~cm}^{-1}$ could come from esters or amide groups [7, 15]. The last one in also present in the Raman spectra of the SYN@VAL and ALI@SYN@VAL samples with little to no shift. Furthermore, the dominant band at $669 \mathrm{~cm}^{-1} / 678 \mathrm{~cm}^{-1}$ from the first two samples is missing. This could be an indication of the induced autism and the local molecular disruption/degradation upon treatment with sodium valproate. 

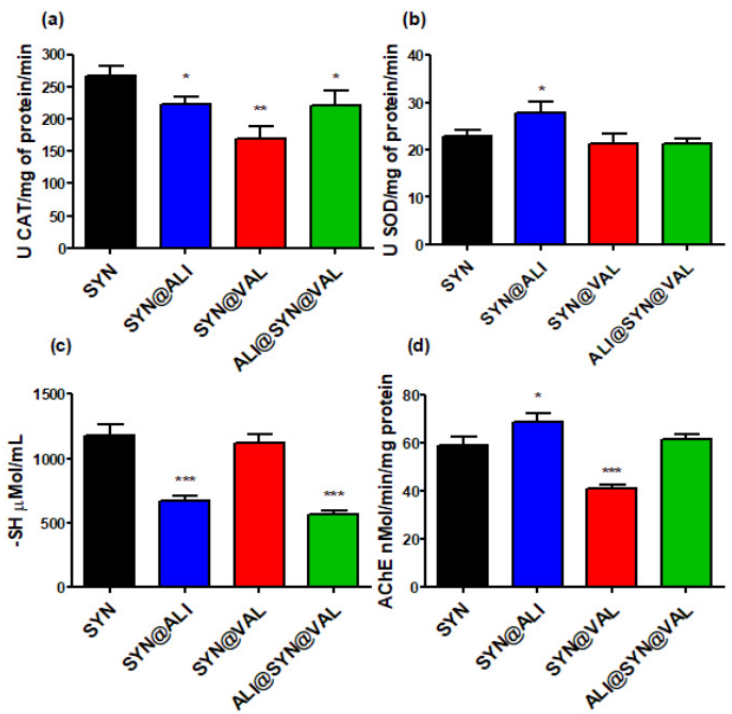

Figure 1. Assessment of CAT (a), SOD (b), -SH (c) and AChE (d) in purified synaptosomes of control (SYN) and experimental groups. Values are expressed as mean \pm SEM. ${ }^{*} P<0.05,{ }^{* *} P<0.01,{ }^{* * *} P<0.001$.

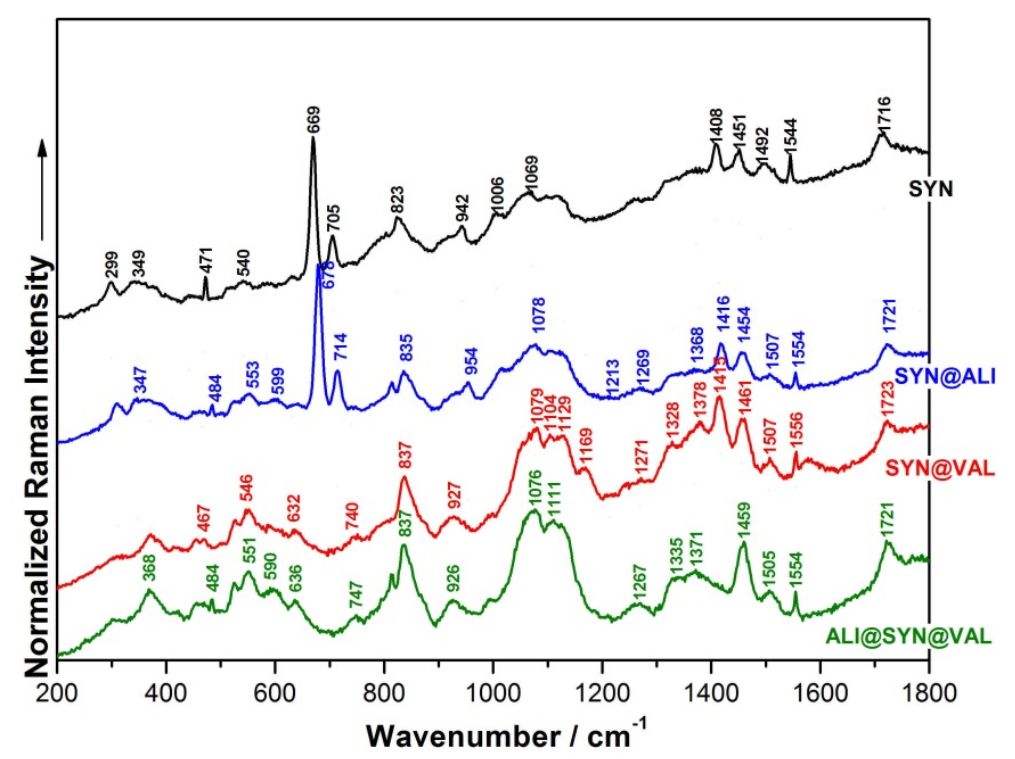

Figure 2. Normalized Raman spectra of the investigated samples. 
VLAD-ALEXANDRU TOMA, ALIA COLNIȚĂ, IOANA BREZEȘTEAN, BOGDAN DUME, IOANA ROMAN, IOAN TURCU

\section{CONCLUSIONS}

Overall, the Raman analysis indicated a similar spectral fingerprint in the case of all four tested samples. The major difference lies in the absence of the $669 \mathrm{~cm}^{-1} / 678 \mathrm{~cm}^{-1}$ in the case of the treated samples with sodium valproate, suggesting an intense structural modification. Based on the spectral features of the investigated samples, one can conclude that the treatment with allicin at the used concentration has no significant influence on the studied synaptosomes. In opposition with these findings, the biochemical measurements depict redox balancing reactions after allicin administration, despite the lack of spectroscopic fingerprint. Further Raman analyses must be done with new different concentrations and volumes.

\section{EXPERIMENTAL SECTION}

\section{Animals}

Adult Wistar female rats (3-month-old) weighing 120-150 g were provided ad libitum access to standard rat chow and water. Animals have been maintained in a light/temperature-controlled room with a light/dark cycle of $12 / 12 \mathrm{~h}$ under $22^{\circ} \mathrm{C}$ constant temperature. Rats were housed $3 /$ cage and all rats in the same cage corresponded to one of the experimental groups. After 3 days of habituation, one male was moved in each cage for the next 5 days. At the end of this period, the males were removed and the first day after male removal was considered as E1 (embryonic day 1). The experimental groups were: Control (SYN), Allicin (SYN@ALI), Valproate (SYN@VAL) and Allicin + Valproate (ALI@SYN@VAL).

\section{Treatment procedures}

In E10 (gestational day 10), the rats were intraperitoneally injected with sodium valproate $550 \mathrm{mg} / \mathrm{kg} \mathrm{b.w}$. in saline solution $0.9 \%$. Concomitantly, the allicin treatment was enteral administered, until the birth of the pups (E20-E21), in all groups without Control. The allicin dose was $2.5 \mathrm{mg} / \mathrm{kg}$ b.w. Animal care and procedures were carried out in accordance to the European Communities Council Directive 2010/63/UE. The procedures of the current work have been approved by the Ethical Committee of Babeș-Bolyai University (IRB no. 2012/03.02.2016).

\section{Neonatal brain sampling and synaptosomes purification}

$12 \mathrm{~h}$ after birth, the corresponding pups of the experimental groups were slightly narcotized with diethyl ether and 3 whole brains/group were dissected out, weighting and placed in Syn-PER buffer (Thermo Fischer, 87793) for synaptosomal purification. The whole tissue was lysed 1:1 w/v with 
Syn-PER buffer and the sample was then centrifugated at $+4^{\circ} \mathrm{C}$, for $10 \mathrm{~min}$, at $1500 \mathrm{rpm}$. The supernatant was collected and spun at $13.500 \mathrm{rpm}$ at $+4^{\circ} \mathrm{C}$ for $20 \mathrm{~min}$. The pellet obtained was the required synaptosomal preparation. For the next measurements, the pellet was resuspended in $5 \mathrm{~mL}$ Syn-PER buffer solution and kept at $-80^{\circ} \mathrm{C}$.

\section{Raman measurements}

Raman measurements were performed on a number of 4 liquid samples: SYN, SYN@VAL, SYN@ALI and ALI@SYN@VAL. The Raman spectra were recorded using a Renishaw InVia Reflex Raman confocal spectrometer with a $532 \mathrm{~nm}$ excitation line. The laser power on the samples was $200 \mathrm{~mW}$. The signal was collected in the range $200-1800 \mathrm{~cm}^{-1}$ using an edge filter $>100 \mathrm{~cm}^{-1}$. The spectral resolution was $1 \mathrm{~cm}^{-1}$. A Leica microscope with a $20 \times$ objective was used to focus and to visualize the liquid samples. The measurements were conducted on $10 \mu \mathrm{L}$.

\section{Biochemical assays}

The isolated synaptosomes were used to assay the activity of CAT, SOD, AChE and the concentration of -SH groups. CAT was assayed by the spectrophotometric measure of the decreasing absorbance of the hydrogen peroxide at $240 \mathrm{~nm}$. SOD was assayed by the method with pyrogallol. The superoxide catalyzes the pyrogallol oxidation and this reaction was measured at $420 \mathrm{~nm}$. The oxidation of the pyrogallol was inversely proportional to the SOD activity. AChE was determined also by a kinetic method using the Ellman reagent (DTNB, dithionitrobenzoic acid) with acetylthiocoline iodide $0.0745 \mathrm{M}$ at $412 \mathrm{~nm}$. Total $-\mathrm{SH}$ groups were colorimetrically assayed with Ellman reagent at $412 \mathrm{~nm}$.

\section{Statistical analyses}

All results are expressed as mean \pm SEM. Comparisons between multiple groups were made using one-way ANOVA followed by Bonferroni's post-hoc test. $P<0.05$ was considered statistically significant and was interpreted as follows: ${ }^{*} P<0.05,{ }^{* *} P<0.01,{ }^{* * *} P<0.001$. Statistical analyses were done using GraphPad Prism 5.

\section{ACKNOWLEDGMENTS}

Work partially supported by the Nucleu Program, Project PN19 350201. 
VLAD-ALEXANDRU TOMA, ALIA COLNIȚĂ, IOANA BREZEȘTEAN,

BOGDAN DUME, IOANA ROMAN, IOAN TURCU

\section{REFERENCES}

1. C. Frisch; K. F. Hüsch; A. Angenstein; W. Kudin; C. E. Kunz; C. Elger Helmestaedter; Epilepsia, 2009, 50, 1432-1441.

2. J. Borlinghaus; F. Albrecht; M. C. H. Gruhlke; I. D. Nwachukwu; A. J. Slusarenko; Molecules, 2014, 19, 12591-12618.

3. C. Hacioglu; F. Kar; G. Kanbak; Med. Sci. Discovery, 2018, 5, 192-197.

4. M. Lasalvia; G. Perna; V. Capozzi; Appl. Spectrosc., 2014, 68, 1123-1131.

5. G. Devitt; K. Howard; A. Mudher; S. Mahajan; ACS Chem. Neurosci., 2018, 9, 404-420.

6. T. D. Payne; A. S. Moody; A. L. Wood; P. A. Pimiento; J. C. Elliott; B. Sharma; Analyst, 2020, 145, 3461-3480.

7. K. Ajito; C. Han; K. Torimitsu; Microsc. Microanal., 2003, 9, 1062-1063.

8. H. Wang; T. S. Tsai; J. Zhao; A. M. D. Lee; B. K. K. Lo; M. Yu; H. Lui; D. I. McLean; H. Zeng; Photodermatol. Photoimmunol. Photomed., 2012, 28, 147152.

9. P. Donfack; M. Rehders; K. Brix; P. Boukamp; A. Materny; J. Raman Spectrosc., 2010, 41, 16-26.

10. Y. Li; Z. N. Wen; L. J. Li; M. L. Li; N. Gao; Y. Z. Guo; J. Raman Spectrosc., 2010, 41, 142-147.

11. H. M. Schipper; C. S. Kwok; S. M. Rosendahl; D. Bandilla; O. Maes; C. Melmed; D. Rabinovitch; D. H. Burns; Biomarkers Med., 2008, 2, 229-238.

12. M. Muratore; Anal. Chim. Acta, 2013, 793, 1-10.

13. D. Sun; X. Chen; ACS Cent. Sci., 2020, 6, 459-460.

14. A. Mizuno; T. Hayashi; K. Tashibu; S. Maraishi; K. Kawauchi; Y. Ozaki; Neurosci. Lett., 1992, 141, 47-52.

15. K. Ajito; K.Torimitsu; Lab. Chip., 2002, 2, 11-14.

16. K. Ajito; C. Han; K. Torimitsu; Anal. Chem., 2004, 76, 2506-2510.

17. V. Toma; A. B. Tigu; A. D. Farcaș; B. Sevastre; M. Taulescu; A. M. Gherman; I. Roman; E. Fischer-Fodor; M. Pârvu; Int. J. Mol. Sci., 2019, 20, 1-18.

18. K. A. Loyd; Biosci. Horiz., 2013, 6, 1-10.

19. A. Rabinkov; T. Miron; D. Mirelman; M. Wilchek; S. Glozman; E. Yavin; L. Weiner; Biochim. Biophys. Acta, 2000, 1499, 144-153.

20. A. L. Christianson; N. Chesler; J. G. Kromberg; Dev. Med. Child. Neurol., 1994, 30, 161-171.

21. M. Kuwagata; T. Ogawa; S. Shioda; T. Nagata; Int. J. Devl. Neuroscience, 2009, 27, 399-405.

22. C. H. Kim; P. Kim; H. S. Go; G. S. Choi; J. H. Park; H. E. Kim; S. J. Jeon; I. C. Pena; S. H. Han; J. H. Cheong; J. H. Ryu; C. Y. Shin; J. Neurochem., 2013, 124, 832-843.

23. S. Kumar; Indian J. Pharmacol., 2015, 47, 444.

24. H. Zhang; P. Wang; Y. Xue; L. Liu; Z. Li; Y. Liu; Tissue and Cell, 2018, 50, 8995. 